\title{
O Estudo do Comportamento Verbal no Brasil
}

\author{
Adriana Pineiro Fidalgo \\ Roberto Alves Banaco \\ Pontifícia Universidade Católica de São Paulo
}

\begin{abstract}
RESUMO - O presente trabalho realizou uma revisão histórica de dissertações de mestrado e teses de doutorado brasileiras sobre comportamento verbal, com base na proposta Skinneriana (1957/1992), produzidas entre 1968 e 2012. Foram investigados: a) tipo de trabalho (dissertação ou tese), b) universidades em que os trabalhos foram defendidos, c) orientadores, d) linha de pesquisa (básica, aplicada ou histórico-conceitual), e) metodologia (descritiva ou experimental), e f) temas de investigação. No total, 177 dissertações e 53 teses sobre comportamento verbal foram identificadas. Os resultados indicam que o estudo do comportamento verbal, no Brasil, estabeleceu-se como programa de pesquisa e cresceu ao longo dos anos.
\end{abstract}

Palavras-chave: comportamento verbal, revisão histórica, análise do comportamento no Brasil

\section{The Study of Verbal Behavior in Brazil}

\begin{abstract}
In the present study a historical review was realized of Brazilian master thesis and doctoral dissertations about verbal behavior, which were based on Skinner' proposals (1957/1992) and carried out from 1968 to 2012. The following aspects were analyzed: a) type of work (thesis or dissertation, b) affiliation of the authors, c) advisor, d) line of research (basic, applied, conceptual or historical), e) methodology (descriptive or experimental), and f) research topics. In total, 177 thesis and 53 dissertations related to verbal behavior were identified. The results indicate that the study of verbal behavior in Brazil has been established as a research program and has grown over the years.
\end{abstract}

Keywords: verbal behavior, historical review, behavior analysis in Brazil

Em 1957, Skinner publicou o livro Verbal Behavior, obra considerada pelo próprio autor como sua principal contribuição à Psicologia. A partir de uma extrapolação de princípios já bem conhecidos da Análise do Comportamento e derivados de rigorosas pesquisas experimentais, Skinner propôs uma interpretação comportamental para o fenômeno tradicionalmente denominado linguagem.

Para evitar vínculos com conceitos utilizados por outros modelos explicativos e ressaltar as diferenças de sua proposta, Skinner atribuiu um novo nome aos fenômenos que pretendia analisar. O termo escolhido por ele foi comportamento verbal. De acordo com o autor, a expressão alude ao comportamento (de um falante individual) modelado e mantido por uma comunidade verbal específica.

Embora submetido às mesmas leis que regem qualquer comportamento operante, o comportamento verbal apresenta algumas particularidades que o tornam exclusivo da espécie humana. Entre elas, está o fato de que ele não age de forma direta ou mecânica sobre o meio: as consequências últimas de uma resposta verbal são mediadas por outro indivíduo, o ouvinte - desde que este tenha passado por um treino especial, o qual lhe permite responder diferencialmente ao comportamento do falante. Anos mais tarde, Skinner (1986) destaca ainda outro aspecto distintivo do comportamento verbal: este deve ser transmitido de geração para geração, tornando-se parte de uma linguagem.

1 Endereço para correspondência: Pontifícia Universidade Católica de São Paulo, Faculdade de Psicologia, Departamento de Métodos e Técnicas em Psicologia, Rua Bartira, 387, Perdizes, São Paulo, SP, Brasil, CEP: 05009-000,E-mail: dripfidalgo@gmail.com
Nos capítulos iniciais de Verbal Behavior, Skinner propôs uma análise científica para o tema linguagem, a qual deveria ser compreendida por meio de análises funcionais capazes de identificar e descrever as relações estabelecidas entre respostas verbais e variáveis ambientais de controle. As unidades de análise propostas foram, dessa forma, relações funcionais (i.e., relações entre condições antecedentes, resposta e consequências) ou contingências de reforçamento. "Ao definir comportamento verbal como comportamento operante mediado, Skinner torna necessariamente a unidade de análise do comportamento verbal uma unidade que envolve uma contingência de reforço" (Andery, 2010, p. 72).

Skinner (1957/1992) intitulou os diferentes tipos de contingências verbais de operantes verbais (a depender das variáveis de controle antecedentes e consequentes presentes). Os operantes verbais descritos pelo autor foram: mando, tato, ecoico, intraverbal, transcrição, textual e autoclítico ${ }^{1}$.

\section{Comportamento Verbal como Área de Pesquisa}

A partir da proposta de Skinner para explicar os fenômenos envolvidos no que tradicionalmente era conhecido como linguagem, numerosas pesquisas foram realizadas. Revisões de literatura (Andery, 2001; Eshleman, 1991; Normand, Fossa, \& Poling, 2002) foram realizadas com o objetivo de

1 Para mais detalhes sobre o conceito de operantes verbais, consultar Skinner (1957/1992) 
caracterizar as pesquisas - sobre comportamento verbal publicadas em diferentes periódicos americanos.

Eshleman (1991) e Normand et al. (2002) apontaram que poucos estudos sobre comportamento verbal foram realizados nas duas primeiras décadas após a publicação do livro Verbal Behavior (1957/1992). No entanto, a partir de 1980, o número de pesquisas na área passou a aumentar de forma sistemática, consolidando um importante campo de pesquisa na Análise do Comportamento.

De acordo com Andery (2001), temas recorrentes nos trabalhos da área são: a) controle operante do comportamento verbal, b) aquisição e interação entre operantes verbais, c) aquisição da linguagem enquanto repertório mais amplo do que a mera aquisição de operantes verbais, d) correspondência entre comportamento verbal e não verbal, e) comportamento governado por regras, f) independência funcional entre operantes verbais, g) solução de problemas, h) questões conceituais sobre a definição de comportamento verbal e de operantes verbais, i) questões metodológicas envolvidas na pesquisa do comportamento verbal, e j) revisão e avaliação dos trabalhos da área.

Um levantamento dos trabalhos nacionais sobre o conceito de comportamento verbal, publicados no período de 1994 a 1998, foi realizado por Moroz, Rubano, Rodrigues e Lucci (2001), com o objetivo de identificar como esse conceito estava sendo investigado pela comunidade brasileira de analistas do comportamento. As autoras utilizaram como fonte de dados 55 periódicos da área e os três primeiros volumes da coleção Sobre Comportamento e Cognição, responsáveis por compilar cinco anos de trabalhos científicos. Enquanto nos periódicos foram encontrados 36 artigos sobre o tema, na coletânea localizaram-se 26 textos, totalizando 62 trabalhos.

Moroz et al. (2001) identificaram cinco áreas de concentração dos trabalhos empíricos: a) processos básicos (51\%), b) educação $(30 \%)$, c) clínica $(13 \%)$, d) desenvolvimento atípico $(3 \%)$, e e) saúde $(3 \%)$. Quanto à metodologia empregada nesses trabalhos, constatou-se predominância de pesquisas experimentais com delineamento de sujeito único, tendo como participantes, sobretudo, estudantes universitários. Entre os trabalhos teórico-conceituais, 68\% priorizaram a análise do Behaviorismo Radical, buscando discutir e aprimorar os pressupostos filosóficos e as definições conceituais, assim como suas implicações práticas. A outra parcela dos trabalhos enfatizou comparações com outras abordagens da Psicologia ou de diferentes áreas do conhecimento, tais como a Linguística.

Moroz et al. (2001) também listaram os principais objetos de estudo das publicações sobre comportamento verbal: a) eventos privados $(30 \%)$, b) comportamento governado por regras $(17 \%), c)$ equivalência de estímulos $(14 \%), d)$ resolução de problemas $(12 \%)$, e) operantes verbais $(11 \%)$, f) modelo explicativo Skinneriano $(11 \%)$, g) relato verbal (3\%), e h) avaliação do repertório verbal (2\%).

À exceção do estudo de Moroz et al. (2001), que analisou apenas um período de cinco anos, não foi encontrado nenhum outro trabalho que tenha proposto a sistematização das pesquisas sobre comportamento verbal no Brasil. Essa tarefa é importante não apenas para identificar o conhecimento produzido e as linhas de pesquisa ou áreas de estudo já consolidadas, mas também para reconhecer aquelas que ainda precisam ser desenvolvidas - pré-requisito para o planejamento e a elaboração de um campo de estudo relevante.

Dessa forma, cabe perguntar: como o conceito de comportamento verbal, tal qual proposto por Skinner (1957/1992), vem sendo estudado por pesquisadores brasileiros desde o início da Análise do Comportamento no país? O presente trabalho teve como objetivo, portanto, realizar uma revisão histórica de dissertações e teses sobre o conceito de comportamento verbal no Brasil. Para isso, analisaram-se os seguintes aspectos gerais dessa área de pesquisa: a) tipos de trabalho (i.e., dissertação ou tese), b) instituições de ensino superior em que os trabalhos foram defendidos, c) orientadores, d) linhas de pesquisa (i.e., básica, aplicada ou histórico-conceitual), e) metodologia (experimental ou descritiva) e f) temas de investigação (e.g., solução de problemas). Ao mesmo tempo, avaliou-se como tais fatores variaram ao longo dos anos. Ainda foram investigadas as publicações (resumos em anais de eventos científicos, capítulos de livro e artigos) resultantes das dissertações e teses analisadas, com o objetivo de estimar a divulgação do conhecimento produzido por tais pesquisadores.

\section{Método}

\section{Documentos}

Os documentos analisados foram dissertações e teses em Análise do Comportamento, produzidas no Brasil, sobre o tema comportamento verbal.

\section{Seleção da fonte}

Para localizar os documentos, consultou-se o Banco de Dados de Dissertações e Teses em Análise do Comportamento no Brasil 1968-2007 (BDTACB) (Micheletto, Guedes, Pereira \& Silva, 2008). Esse banco foi elaborado por meio de uma pesquisa extensa realizada: 1) nos sites ou bibliotecas das universidades com cursos de pós-graduação em Análise do Comportamento [e.g. USP (1971), UnB (1974), UFSCar (1978)] e de outras universidades conhecidas por reunir pesquisadores em Análise do Comportamento; 2) no banco de dissertações e teses da CAPES (Coordenação de Aperfeiçoamento de Pessoal de Nível Superior); 3 ) nos Currículos Lattes dos orientadores até então localizados, nos quais foram identificadas dissertações e teses por eles orientadas, mas não detectadas no levantamento inicial; e 4) nos Currículos Lattes de todos os orientandos dos primeiros orientadores, destinada a verificar eventuais trabalhos orientados pelos novos pesquisadores. O levantamento completo das dissertações e teses foi realizado por três gerações de pesquisadores. O BDTACB, até 2007, reunia dados de 789 dissertações e 221 teses (1010 trabalhos, no total). O primeiro deles data de 1968; o último, até a realização deste estudo, 
de $2007^{2}$. Entre as informações listadas para cada trabalho inserido nesse banco de dados estão: a) autor, b) título, c) ano da defesa, d) orientador, e) instituição de ensino superior, f) tipo de trabalho (i.e., dissertação ou tese), e g) linha de pesquisa (i.e., básica, aplicada ou histórico-conceitual). Apenas uma parcela dos estudos apresenta informações sobre a) filiação atual do autor, b) filiação atual do orientador, c) coorientador, d) filiação atual do co-orientador, e) curso, f) palavras-chave, e g) Resumo.

Uma vez que o BDTACB não continha os resumos de todas as dissertações e teses presentes no banco, procedeu-se à localização dessas informações antes de se iniciar a coleta de dados. A princípio, foram identificados os trabalhos em que o campo Resumo estava em branco. Em seguida, os dados ausentes foram procurados em meios eletrônicos (i.e., sites dos programas de pós-graduação, banco de dissertações e teses da CAPES e arquivos no formato $p d f$ disponibilizados na internet) ou nas bibliotecas da PUC-SP e da USP. Por fim, acrescentaram-se as informações encontradas ao BDTACB.

Após a atualização do banco de dados, dos 381 trabalhos inicialmente sem Resumo, 145 ainda permaneceram dessa forma. Para tais pesquisas, apenas o título foi utilizado na busca das palavras selecionadas para identificar estudos sobre comportamento verbal, conforme descrito a seguir.

\section{Seleção dos documentos: dissertações e teses sobre comportamento verbal}

A fim de localizar as dissertações e teses brasileiras relacionadas ao conceito de comportamento verbal, inicialmente foram definidas 15 palavras de busca: "verbal", "verbais", "verbalizações", "linguagem", "mando", "tato", "tacto", "ecoico", "intraverbal", "transcrição", "textual", "ditado", "cópia", "autoclítico" e "audiência". Para ser incluído na presente pesquisa, o estudo deveria apresentar pelo menos uma dessas palavras em seu título ou no Resumo.

Para alguns autores (Hall, 1998; Hall \& Chase, 1991; Horne \& Lowe, 1997; Wulfert, Dougher, \& Greenway, 1991), a definição de linguagem ou de comportamento verbal deveria envolver o paradigma da equivalência de estímulos. No entanto, uma vez que a presente pesquisa visava a análise de estudos sobre comportamento verbal à luz do Behaviorismo Radical de Skinner, não foram incluídas palavras de busca relacionadas à equivalência de estímulos, pois Skinner não se referiu a esse conceito ao longo de sua vasta obra.

De modo semelhante, palavras de busca relacionadas ao conceito de comportamento governado por regras também não foram incluídas nesta pesquisa. Isso porque, adotou-se como referência para a definição de temas ligados ao conceito de comportamento verbal o livro Verbal Behavior, publicado em 1957, enquanto a expressão comportamento governado por regras surgiu pela primeira vez somente em 1963, em texto de Skinner publicado seis anos após o lançamento de Verbal Behavior (Mendonça, 2010). Outros conceitos tratados pelo autor no âmbito do comportamento verbal, como solução de problemas (Skinner, 1966/1969),

2 Para informações mais detalhadas sobre o BDTACB, ver Fidalgo (2011). também não foram incluídos entre as palavras de busca por motivos semelhantes.

\section{Atualização parcial do BDTACB: localização de trabalhos sobre comportamento verbal defendidos no período de 2008 a 2012}

Uma vez que o BDTACB contém dissertações e teses defendidas até o ano de 2007, a autora do presente estudo realizou um levantamento bibliográfico com o objetivo de identificar dissertações e teses sobre comportamento verbal defendidas no período de 2008 a 2012.

Após a localização dos trabalhos sobre comportamento verbal no BDTACB (por meio dos métodos descritos na sessão "Seleção dos Documentos: Dissertações e Teses sobre Comportamento Verbal"), foram identificados as seis Universidades que produziram o maior número de trabalhos na área (i.e., USP, PUC-SP, UFPA, UnB, UFSCar e UCG). Os trabalhos defendidos nas seis universidades, juntos, correspondem a $95 \%$ do total de estudos na área. Em seguida, foi realizada uma busca nas bibliotecas digitais de "dissertações e teses dessas instituições".

Os trabalhos defendidos entre os anos de 2008 e 2012, que se enquadraram nos critérios de seleção explicitados na seção "Seleção dos Documentos: Dissertações e Teses sobre Comportamento Verbal”, foram selecionados para análise.

\section{Registro e categorização dos dados}

Após a identificação das dissertações e teses brasileiras sobre comportamento verbal, um novo banco de dados foi criado com o programa Microsoft Office Excel 2007. Após a leitura integral dos resumos das dissertações e das teses, com base nas propostas de categorização de Andery (2001), Moroz et al. (2001) e Micheletto, Guedes, César, \& Pereira (2010), foram elaboradas as seguintes categorias ${ }^{3}: 1$ ) autor do trabalho; 2) título do trabalho; 3 ) ano da defesa; 4) tipo de trabalho (i.e., dissertação ou tese); 5) instituição de ensino superior; 6) orientador; 7) linha de pesquisa: pesquisa básica (trabalhos destinados a investigar experimentalmente conceitos e processos comportamentais básicos, fundamentados no referencial teórico da Análise do Comportamento), pesquisa aplicada (trabalhos voltados a analisar problemas originados de uma demanda social concreta, cujos resultados buscam atender às demandas) ou pesquisa histórico-conceitual (trabalhos destinados a avaliar o desenvolvimento histórico e as bases epistemológicas, metodológicas e conceituais do Behaviorismo Radical e da Análise do Comportamento), conforme definidas por Micheletto et al. (2010); 8) metodologia (experimental ou descritiva); 9) temas (diferentes subprogramas de pesquisa sobre comportamento verbal ou áreas distintas de interesse sobre o tema [Andery, 2001]): controle operante do comportamento verbal; aquisição e interação entre operantes verbais; solução de problemas; relações entre

3 Para definição mais detalhada de cada campo elaborado pela pesquisadora, ver Fidalgo (2011). 
comportamento verbal e não verbal; comportamento verbal e noção de subjetividade; metodologia utilizada no estudo do comportamento verbal; estudo de relações que emergem sem reforçamento direto; análise teórico-conceitual; levantamento de hipóteses funcionais; análise de conteúdo do relato verbal; metodologia utilizada no estudo do comportamento verbal $)^{4}$.

\section{Publicação de dissertações e teses sobre comportamento verbal}

A localização das publicações decorrentes das dissertações e teses sobre comportamento verbal foi realizada da seguinte maneira:

1. Procurou-se o Currículo Lattes de todos os autores de trabalhos sobre comportamento verbal identificados.

2. Em cada currículo localizado, analisou-se a produção bibliográfica do autor.

3. As publicações resultantes das dissertações e teses incluíam: 1) resumos em anais de congressos, publicados após o ano de defesa da dissertação e/ou da tese, cujo título indicasse relação entre a publicação e o trabalho de mestrado e/ou de doutorado; 2) capítulos de livros; e 3) artigos completos em periódicos.

\section{Concordância entre juízes}

Para as categorias copiadas do BDTACB (i.e., Autor, Título, Ano, Orientador, Instituição, Tipo de trabalho e Linha de pesquisa), não foi realizada a concordância entre juízes.

Para as demais categorias elaboradas pela pesquisadora (i.e., Área de aplicação, Conceitos da Análise do Comportamento e Temas), a concordância entre juízes foi realizada da seguinte maneira:

1. Para cada categoria, foram selecionados, pela autora do presente trabalho, 20\% dos trabalhos passíveis classificação na categoria em questão. A escolha dos trabalhos foi feita de forma aleatória - por exemplo, a pesquisadora pedia para que o juiz escolhesse um número de 1 a 100 e, assim, a pesquisa cujo número era correspondente ao número escolhido pelo juiz era selecionada para análise.

2. A pesquisadora (primeira juíza) classificou os trabalhos segundo as descrições de cada categoria.

3. Após ter acesso às descrições das categorias, um segundo juiz (pesquisador com experiência em Análise do Comportamento) classificou os mesmos trabalhos analisados pela pesquisadora.

4. Quando o acordo entre juízes a respeito da classificação dos trabalhos em uma determinada categoria era equivalente a $80 \%$, considerava-se satisfatória a definição/

4 Apesar do presente trabalho não ter tido como objetivo a investigação de temas não relacionados ao livro Verbal Behavior (1957) (e.g. equivalência de estímulos, regras, e solução de problemas), algumas das pesquisas selecionadas na coleta de dados investigaram esses tópicos. Dessa forma, foi necessária a criação de categorias de análise que considerassem esses trabalhos. Uma análise pormenorizada dessa questão será posteriormente realizada nas seções de Resultados e Discussão e de Considerações Finais. descrição da categoria analisada. A partir daí, os demais trabalhos foram classificados somente pela pesquisadora.

5. Quando o acordo entre juízes a respeito da classificação dos trabalhos em uma determinada categoria era inferior a $80 \%$, os juízes discutiam a definição da categoria em questão e a pesquisadora reformulava a descrição. $\mathrm{Na}$ sequência, uma nova classificação era realizada e avaliada pelos pesquisadores.

Este processo se estendeu até ser alcançado $80 \%$ de concordância entre os juízes nas diversas categorias adotadas ao analisar as dissertações e teses incluídas na presente pesquisa.

\section{Resultados e Discussão}

Dos 1010 trabalhos reunidos no BDTACB (de 1968 a 2007), 184 deles (143 dissertações e 41 teses) versaram sobre comportamento verbal [ver referências completas em Fidalgo (2011)]. O levantamento bibliográfico, que abrangeu o período de 2008 a 2012, localizou 46 novos trabalhos sobre o tema (36 dissertações e 12 teses). Dessa forma, o número total de pesquisas sobre comportamento verbal analisadas no presente estudo foi de 230, sendo 177 dissertações e 53 teses.

A Figura 1 mostra como o número dessas pesquisas variou ao longo dos anos, entre os anos de 1968 e 2012. Para facilitar a compreensão de como a abertura de programas de pós-graduação na área impactou a produção de trabalhos sobre comportamento verbal, foram indicados os anos em que os sete programas de pós-graduação que mais produziram trabalhos sobre esse conceito apresentaram suas primeiras defesas de dissertações de mestrado ou teses de doutorado (linhas tracejadas). Como a defesa de uma dissertação e/ou tese só pode ser realizada dois anos após a criação de um programa de pós-graduação, as linhas tracejadas foram colocadas dois anos após a abertura dos programas em questão, a saber: Programa de Pós-Graduação em Psicologia Experimental da Universidade de São Paulo (USP) (1971); Programa de Pós-Graduação em Psicologia (UnB) (1974)5; Programa de Pós-Graduação em Educação Especial da Universidade Federal de São Carlos (UFSCar) (1978); Programa de Pós-Graduação em Teoria e Pesquisa do Comportamento da Universidade Federal do Pará (UFPA) (1987); Programa de Estudos Pós-Graduados em Psicologia Experimental: Análise do Comportamento da Pontifícia Universidade Católica de São Paulo (PUC-SP) (1999); Programa de Pós-Graduação Stricto Senso da Universidade Católica de Goiás (UCG) (1999); Programa de Pós-Graduação em Análise do Comportamento da Universidade Estadual de Londrina (UEL) (2005).

O primeiro estudo identificado (Engelmann, 1969) foi uma dissertação de mestrado, elaborada na USP, sobre comportamento verbal como objeto de estudo da Psicologia, orientada por Carolina Bori. De 1969 a 1979, a produção de trabalhos sobre o tema foi limitada no Brasil: apenas seis pesquisas, todas orientadas por Carolina Bori, na USP.

5 Esse programa foi subdividido, no período 2005-2007, em quatro programas de pós-graduação, dentre eles o Programa de Pós-Graduação em Ciências do Comportamento, no qual estao inseridos os analistas do comportamento. 


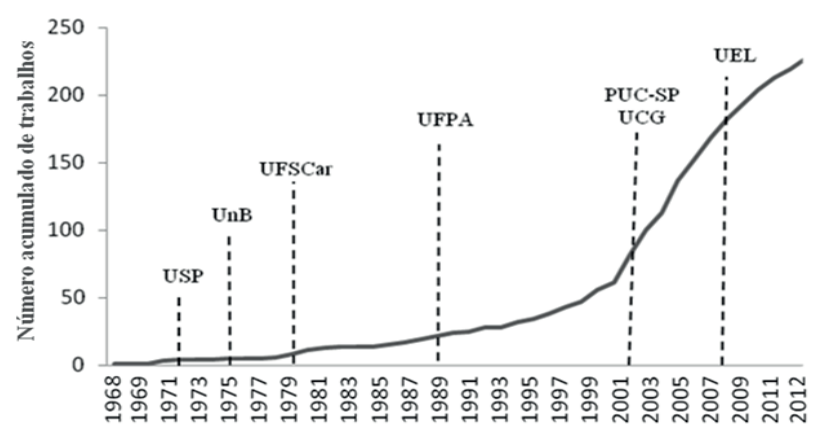

Figura 1. Número acumulado de trabalhos sobre comportamento verbal ao longo dos anos.

De 1979 ao início da década de 1990, observa-se um pequeno aumento no número de estudos sobre comportamento verbal. Até 1990, tal crescimento não pode ser atribuído à criação de programas de pós-graduação em outras universidades - uma vez que, dos 28 trabalhos identificados até esse ano, apenas dois não foram produzidos na USP. Esse aumento parece estar mais relacionado à ampliação do número de orientadores na USP. Entre eles, Maria Amélia Matos, Rachel Kerbauy e Luis Cláudio Figueiredo.

Outro fator que pode ter contribuído para o crescimento do número de pesquisas sobre comportamento verbal nessa época foi a tradução do livro Verbal Behavior para o português, em 1978, iniciativa que aproximou os pesquisadores brasileiros da nova área de investigação proposta por Skinner.

Durante a década de 1990, a orientação de trabalhos sobre o tema se estendeu a outras universidades, como a UnB, a UFPA e a UFSCar. Interessante notar que, apesar de os programas de pós-graduação dessas três universidades terem sido criados na década de 1970, somente a partir dos anos 1990 elas começaram a produzir estudos nesse novo campo de pesquisa.

Ao final da década de 1990, com a criação de novos cursos de pós-graduação em Análise do Comportamento (em especial, os cursos da PUC-SP e da UCG), o número de trabalhos sobre comportamento verbal aumentou significativamente. É possível afirmar também que a produção de trabalhos sobre comportamento verbal tem aumentado de forma sistemática, principalmente a partir dos anos 1980, indicando a consolidação desse campo de pesquisa no país.

Tipo de trabalho e Instituição de Ensino Superior

Quanto ao tipo de trabalho elaborado, 77\% dos estudos identificados foram dissertações de mestrado e $23 \%$, teses de doutorado.

A Figura 2 apresenta o número acumulado de pesquisas realizadas pelas seis universidades brasileiras com maior quantidade de trabalhos na área. A PUC-SP foi a universidade com o maior número de trabalhos sobre comportamento verbal (57), sendo 54 dissertações e três teses. Em seguida, apareceu a USP, com 51 pesquisas (26 dissertações e 25 teses). A UFPA e a UFSCar apareceram em terceiro lugar, com 33 trabalhos cada uma (a UFPA produziu 29 dissertações e quatro teses, e a UFSCar, 21 dissertações e 12 teses). A UnB foi a quarta colocada, com 31 pesquisas (22 dissertações e

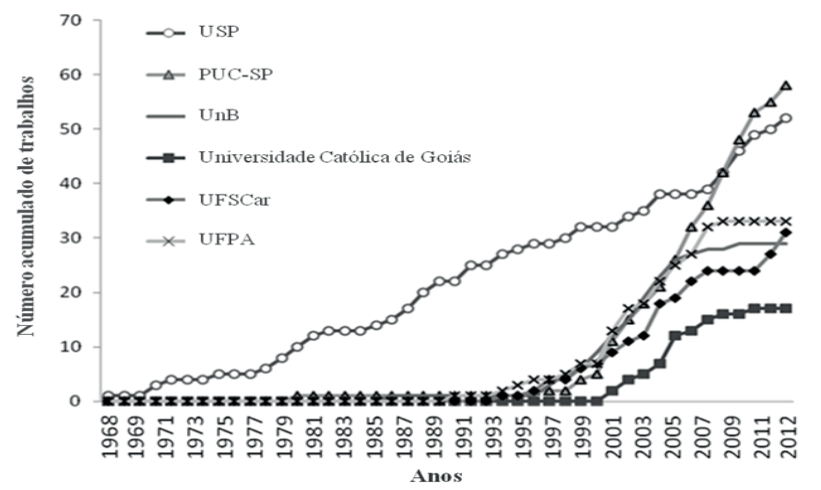

Figura 2. Número acumulado de trabalhos produzidos pelas seis universidades brasileiras com maior volume de pesquisas sobre comportamento verval ao longo dos anos

nove teses), seguida pela UCG, com 17 trabalhos na área (todos dissertações de mestrado). Quatro instituições adicionais também produziram trabalhos sobre comportamento verbal no período investigado (dados não mostrados na Figura 2): a UEL e o Mackenzie (três dissertações cada), a UFSC e a UNESP (uma dissertação cada).

A USP foi a principal instituição responsável pela elaboração de estudos sobre comportamento verbal até o início da década de 1990. Diferentemente das outras universidades, a PUC-SP e a UCG apresentaram um grande número de dissertações e teses sobre comportamento verbal defendido logo após a criação de seus programas de pós-graduação, contribuindo decisivamente para ampliar os estudos na área a partir do ano de sua abertura.

\section{Orientadores}

A pesquisadora com o maior número de trabalhos orientados sobre comportamento verbal foi Carolina Bori, totalizando 17 pesquisas (oito dissertações e nove teses). $\mathrm{Na}$ sequência, aparecem Maria Amália Andery (15 dissertações), Maria Martha Hübner (13 orientações: sete dissertações e seis teses), Roberto Banaco (12 dissertações), Deisy das Graças de Souza (11 orientações: nove dissertações e duas teses), Emmanuel Tourinho (11 orientações: nove dissertações e duas teses), Júlio de Rose (10 orientações: seis dissertações e quatro teses), Maria Amélia Matos (nove orientações: duas dissertações e sete teses) e, por fim, Antônio Ribeiro (oito orientações: seis dissertações e duas teses).

\section{Linhas de pesquisa}

Em relação à linha de pesquisa em que os trabalhos foram realizados, constatou-se que, dos 230 estudos sobre comportamento verbal, 127 eram pesquisas aplicadas (55\%), 85 , pesquisas básicas $(37 \%)$ e 18 , pesquisas histórico-conceituais $(8 \%)$.

A Figura 3 mostra a variação na frequência de trabalhos realizados nas diferentes linhas de pesquisa ao longo dos anos. 


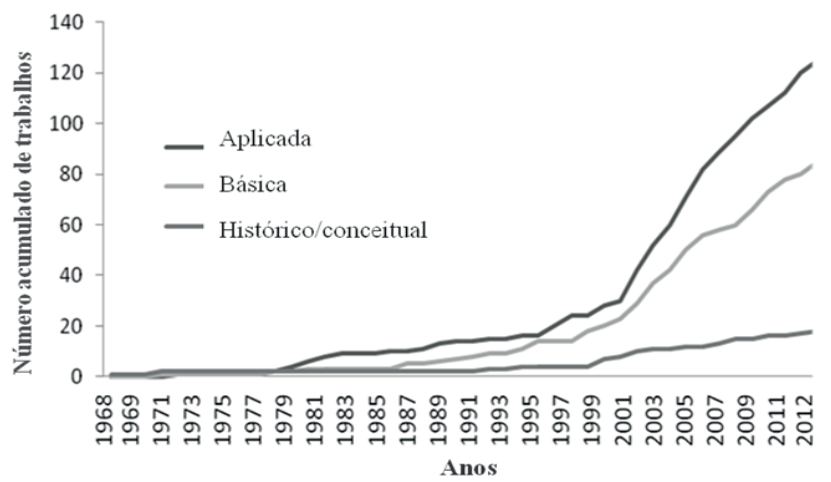

Figura 3. Número acumulado de trabalhos nas diferentes linhas de pesquisa por ano.

Ao contrário da produção total de dissertações e teses em Análise do Comportamento no Brasil, em que as pesquisas básicas prevaleceram até o final da década de 1990 (Micheletto et al., 2010), a maioria dos trabalhos sobre comportamento verbal consistiu de pesquisas aplicadas, realizadas do início ao fim do período analisado no presente estudo. Até 1979, a produção de trabalhos nas três linhas de pesquisa se deu de forma semelhante. No entanto, a partir de 1980, o número de pesquisas aplicadas começou a crescer, distanciando-se do número de trabalhos nas linhas restantes. O número de pesquisas básicas passou a crescer apenas 10 anos depois (a partir da década de 90), e as pesquisas histórico-conceituais, 20 anos depois. A partir do ano 2000, vale acrescentar, trabalhos nas três linhas de pesquisa aumentaram consideravelmente de frequência, acompanhando o crescimento geral de estudos brasileiros sobre comportamento verbal observado a partir dessa data.

A maioria dos trabalhos aplicados (49\%) foi realizada na área de educação. Um quarto das pesquisas aplicadas (25\%) foi conduzido na área clínica. Apenas 10\% dos trabalhos aplicados foram realizados na área da saúde. As demais pesquisas aplicadas (16\%) foram conduzidas em diferentes áreas de aplicação, tais como esporte, mídia e treino de habilidades básicas necessárias à comunicação.

O principal conceito abordado pelas pesquisas básicas ao estudar o comportamento verbal foi o de contingências de reforçamento $(27 \%)$. Outro conceito recorrente entre as pesquisas básicas foi o de comportamento governado por regras (24\%). Embora excluído das palavras de busca utilizadas no presente estudo para localizar os trabalhos sobre comportamento verbal, tal conceito foi identificado em diversas pesquisas selecionadas. Presente em 16\% das pesquisas básicas sobre comportamento verbal, o conceito de equivalência de estímulos e os termos relacionados a ele também foram excluídos das palavras de busca adotadas no presente trabalho. Contudo, dado o número significativo de pesquisas que o abordaram, tornou-se evidente a relevância e a inserção da proposta de Sidman e Tailby (1982) nessa área de pesquisa. Em menor frequência, outros conceitos também foram mencionados nos trabalhos básicos, a saber: controle aversivo, solução de problemas, operação motivadora, variabilidade, autocontrole, eventos privados e metacontingências.

Os trabalhos de natureza histórico-conceitual se dedicaram a: a) investigar questões teóricas e filosóficas do Beha- viorismo Radical (cinco pesquisas), b) estudar conceitos da Análise do Comportamento (oito pesquisas), e c) comparar o Behaviorismo Radical e/ou a Análise do Comportamento às outras áreas do conhecimento (duas pesquisas). O número relativamente limitado de pesquisas histórico-conceituais sugere que analistas do comportamento brasileiros ainda não debruçaram sobre a própria produção científica no campo do comportamento verbal. De acordo com Morris, Todd, Midgley, Scheneider e Johnson (1985), a condução de trabalhos nessa linha de pesquisa - responsável por colecionar, organizar e examinar os estudos até então realizados em uma disciplina - indicaria o crescimento e o amadurecimento da área.

\section{Metodologia}

Das 230 pesquisas sobre comportamento verbal, 64\% utilizaram metodologia experimental e 36\%, metodologia descritiva. Dentre as pesquisas experimentais, $97 \%$ adotaram o delineamento de sujeito único, tendo o restante utilizado delineamento de grupo. Os dados - opostos aos achados de Moroz et al. (2001), que apontaram a prevalência de estudos teóricos ao analisar artigos nacionais sobre o tema - indicam que as dissertações e teses brasileiras sobre comportamento verbal têm seguido o modelo de metodologia proposto pela Análise do Comportamento, que privilegia experimentação e delineamento de sujeito único.

\section{Temas de pesquisa}

Os temas propostos no presente estudo variaram segundo o tipo de pesquisa realizada: experimental ou descritiva.

Tabela 1. Número Total e Porcentagem dos Temas de Investigação das Pesquisas Experimentais

\begin{tabular}{lcc}
\multicolumn{1}{c}{ Temas } & N & \% \\
\hline Equivalência de estímulos & 40 & $26 \%$ \\
Controle operante do comportamento verbal & 31 & $21 \%$ \\
$\begin{array}{l}\text { Comportamento governado por regras } \\
\text { Correspondência entre comportamento }\end{array}$ & 29 & $19 \%$ \\
$\begin{array}{l}\text { verbal e não verbal } \\
\text { Independência funcional entre operantes }\end{array}$ & 9 & $15 \%$ \\
$\begin{array}{l}\text { verbais } \\
\text { Aquisição, manutenção e generalização de } \\
\text { operantes verbais }\end{array}$ & 9 & $5 \%$ \\
$\begin{array}{l}\text { Comportamento verbal e noção de } \\
\text { subjetividade }\end{array}$ & 4 & $3 \%$ \\
$\begin{array}{l}\text { Solução de problemas } \\
\text { Metodologia utilizada no estudo do } \\
\text { comportamento verbal }\end{array}$ & 4 & $3 \%$ \\
$\begin{array}{l}\text { Controle do comportamento verbal sobre o } \\
\text { comportamento não verbal correspondente }\end{array}$ & 2 & $1 \%$ \\
\hline$\quad$ Total & $\mathbf{1 5 4}$ & - \\
\hline
\end{tabular}




\section{Pesquisas experimentais}

Os principais temas investigados nas pesquisas experimentais foram agrupados, no presente trabalho, de acordo com as categorias propostas por Andery (2001), conforme indicado na Tabela 1.

O estudo de relações verbais emergentes, ou paradigma da equivalência, foi o tema mais investigado pelas pesquisas experimentais $(26 \%)$. Boa parte dos trabalhos avaliou os processos envolvidos na aquisição de leitura com compreensão e na emergência de relações numéricas. O segundo tema prevalente foi o controle operante do comportamento verbal (21\%). Esses estudos analisaram o modo como processos operantes básicos se aplicam ao comportamento verbal. Entre outras, destacaram-se as pesquisas voltadas a estudar: a) o efeito de diferentes contingências de reforçamento sobre respostas verbais, b) o estabelecimento da audiência como estímulo discriminativo, e c) o efeito da punição sobre o comportamento verbal.

Comportamento governado por regras representou o terceiro tema mais abordado pelas pesquisas experimentais $(19 \%)$, elegendo como objetivo principal investigar as diferenças entre o controle estabelecido por contingências de reforço e o controle exercido por regras e instruções. $\mathrm{Na}$ sequência, figuraram as pesquisas destinadas a avaliar a correspondência entre comportamento verbal e comportamento não verbal (15\%), cuja finalidade principal era compreender a maneira como tal correspondência se estabelece e identificar as variáveis ambientais capazes de distorcê-la. A independência funcional entre operantes verbais, por sua vez, foi o tema investigado por $5 \%$ das pesquisas experimentais. Estes trabalhos abordaram especialmente a independência entre a aquisição de mandos e de tatos. Outros 5\% dos estudos analisaram a aquisição e a manutenção de operantes verbais, com destaque para os processos envolvidos no treino de aquisição e generalização desses operantes.

Cabe citar, ainda, outros temas identificados: a) noção de subjetividade $(3 \%)$, representada por trabalhos interessados em investigar os processos envolvidos no ensino de descrição de eventos privados; b) solução de problemas (3\%); c) questões metodológicas $(2 \%)$, com pesquisas orientadas a avaliar ou desenvolver procedimentos de categorização e registro de respostas verbais; e d) controle do comportamento verbal sobre o comportamento não verbal correspondente (1\%).

De novo, vale fazer uma ressalva: o presente trabalho não teve por objetivo analisar de forma específica os campos de pesquisa sobre comportamento governado por regras e equivalência de estímulos. Contudo, embora a busca de estudos sobre comportamento verbal não tenha visado à identificação de trabalhos a respeito desses conceitos, os mesmos foram detectados na amostra final. Ainda assim, os resultados aqui descritos não devem ser considerados representativos dos campos de pesquisa sobre comportamento governado por regras e equivalência de estímulos, mas apenas como indícios de que seu estudo relaciona-se intimamente com a área de investigação do comportamento verbal.

\section{Pesquisas descritivas}

Foram identificados quatro temas de investigação nas pesquisas descritivas. A Tabela 2 apresenta o número e a porcentagem de trabalhos em cada tema. A maior parte das
Tabela 2. Número Total e Porcentagem dos Temas de Investigação das Pesquisas Descritivas

\begin{tabular}{lcc}
\hline \multicolumn{1}{c}{ Temas das pesquisas descritivas } & N & \% \\
\hline Hipótese funcional & 43 & $57 \%$ \\
Análise teórico-conceitual & 16 & $21 \%$ \\
Metodologia utilizada no estudo do & 9 & $12 \%$ \\
comportamento verbal & & \\
Análise de conteúdo & 7 & $10 \%$ \\
Total & 75 & - \\
\hline \multicolumn{2}{c}{ Total } \\
\hline
\end{tabular}

pesquisas descritivas (57\%) buscou elaborar hipóteses funcionais sobre variáveis de controle possivelmente envolvidas no comportamento verbal. Por exemplo, a observação de sessões terapêuticas permitiu descrever relações de controle entre as respostas verbais do terapeuta e as respostas verbais e não verbais do cliente. De maneira semelhante, alguns estudos avaliaram a interação verbal entre professores e alunos no contexto escolar.

Análises teórico-conceituais foram realizadas em 21\% das pesquisas descritivas. Destas, $77 \%$ analisaram a evolução de conceitos do Behaviorismo Radical, com a finalidade de sistematizar e aprimorar a base conceitual da Análise do Comportamento. Os demais trabalhos (23\%) estabeleceram comparações (i.e., aproximações ou contraposições) com os pressupostos de outras abordagens ou áreas do conhecimento, relacionadas principalmente às formulações tradicionais sobre a linguagem. Moroz et al. (2001) também indicaram a predominância de estudos teórico-conceituais voltados a discutir os pressupostos filosóficos da Análise do Comportamento $(68 \%)$. No trabalho realizado pelas autoras, uma porcentagem menor de estudos (32\%) também realizou comparações com outras abordagens ou áreas do conhecimento.

Trabalhos destinados a avaliar a metodologia adotada no estudo do comportamento verbal responderam por $12 \%$ do total de pesquisas descritivas. Entre elas, destacaram-se os estudos orientados à análise de procedimentos de categorização e registro de respostas verbais. Uma parcela menor dos trabalhos descritivos (10\%) investigou ainda o conteúdo do comportamento verbal, baseando-se na topografia de respostas verbais.

\section{Publicação de dissertações e teses sobre comportamento verbal}

A análise do Currículo Lattes dos pesquisadores sobre comportamento verbal permitiu identificar quais dissertações e teses brasileiras foram publicadas. Os meios de publicação considerados foram 1) resumos em anais de eventos científicos, 2) artigos de periódicos científicos, e 3) capítulos de livros.

Dos 230 autores dos trabalhos analisados nesta pesquisa, localizou-se o Currículo Lattes de 183 deles. Destes, 81 (44\%) publicaram as dissertações ou teses em pelos menos um dos meios citados anteriormente (e.g., artigos em periódicos científicos). Quarenta e um publicaram os estudos apenas na forma de Resumo em anais de eventos, 21 somente 
como artigo e quatro exclusivamente como capítulo de livro. Alguns autores divulgaram os trabalhos em mais de um meio: oito deles em resumos e artigos, seis em resumos e capítulos de livro e um deles em artigo e capítulo de livro. No total, foram identificados 50 resumos em anais de eventos científicos, 24 artigos e 13 capítulos de livros.

A análise de publicações derivadas de dissertações e teses sobre comportamento verbal é relevante, pois indica o impacto do conhecimento produzido nessas pesquisas sobre a comunidade de analistas do comportamento e de pesquisadores de outras abordagens.

Uma vez que apenas menos da metade (44\%) dos pesquisadores da área publicou seus trabalhos, o acesso aos demais estudos restringe-se aos exemplares presentes nas bibliotecas das universidades ou às versões eletrônicas disponibilizadas nos sites das instituições. Neste último caso, porém, somente um número limitado de universidades permite acesso ao trabalho integral, o que dificulta o intercâmbio de conhecimento entre as diferentes regiões do país. Considerando a eficiência dos periódicos científicos no que se refere à divulgação de conhecimento, e que essa divulgação é essencial para o desenvolvimento de uma área de investigação, faz-se urgente que os pesquisadores finalizem o processo de produção de conhecimento com a publicação de seus trabalhos.

Ainda é importante considerar os limites do material analisado (i.e., Currículo Lattes dos autores de dissertações e teses sobre comportamento verbal) e suas implicações na análise dos dados aqui apresentados. Não são todos os pesquisadores que possuem Currículo Lattes ou que o atualizam periodicamente. Dessa forma, o número de publicações decorrentes de dissertações e teses pode ser ainda maior do que o constatado no presente trabalho.

\section{Considerações finais}

Alguns autores da Análise do Comportamento (Donahoe, 1998; Drash \& Tudor, 1991; Leigland, 1998) têm afirmado que a proposta de Skinner (1957/1992) para o estudo do comportamento verbal não gerou um campo de pesquisa expressivo. Os resultados do presente trabalho, porém, apontam que o estudo do comportamento verbal no Brasil, de acordo com a proposta conceitual de Skinner, estabeleceu-se como programa de pesquisa (i.e. conjunto de pesquisas que tem como objetivo mapear e gradativa e paulatinamente abarcar um tema de investigação) e cresceu ao longo dos anos.

Carolina Bori pode ser apontada como figura central tanto na implantação quanto na condução do estudo do comportamento verbal no Brasil. Outros pesquisadores que merecem destaque pelo grande número de trabalhos orientados neste campo de pesquisa são: Deisy das Graças Sousa, Emmanuel Tourinho, Maria Amália Andery, Roberto Banaco, Maria Amélia Matos, Antônio Ribeiro e Júlio de Rose.

A produção de trabalhos sobre comportamento verbal no país é composta principalmente por dissertações de mestrado conduzidas na linha de pesquisa aplicada. Pôde-se identificar uma grande variedade de temas investigados nas diferentes universidades. Os temas mais frequentes foram: controle operante do comportamento verbal, comportamento governado por regras, equivalência de estímulos, correspondência entre comportamento verbal e comportamento não verbal e, por fim, elaboração de hipóteses funcionais a respeito das possíveis variáveis de controle envolvidas no comportamento verbal.

Como já discutido anteriormente, o presente trabalho não teve por objetivo caracterizar os campos de pesquisa de equivalência de estímulos e de comportamento governado por regras, já que ambos não foram abordados por Skinner no livro Verbal Behavior (1957/1992). No entanto, o grande número de dissertações e teses sobre esses temas evidencia uma relação estreita entre eles e o estudo do comportamento verbal. Por esse motivo, sugere-se que trabalhos futuros investiguem de forma mais cuidadosa aspectos específicos dessas três áreas de pesquisa e analisem criteriosamente a forma como elas interagem.

O presente trabalho mostrou ainda que apenas metade das dissertações e teses sobre comportamento verbal analisadas foi publicada. O impacto do conhecimento produzido sobre a comunidade de pesquisadores e profissionais da psicologia ou de outras áreas do conhecimento é potencializado pela divulgação em meios mais acessíveis à população geral, como periódicos científicos (impressos ou eletrônicos), livros e apresentações em congressos e afins.

Admitindo a importância de os trabalhos serem divulgados em diferentes meios científicos, sugere-se que autores de dissertações e teses ampliem a publicação de seus trabalhos, assim como criem ou atualizem seus currículos Lattes - ferramenta que auxilia na localização dos trabalhos produzidos. Propõe-se, ainda, que pesquisas futuras investiguem como o estudo do comportamento verbal vem sendo abordado nos meios de divulgação (e.g. periódicos científicos, livros, coletâneas, anais, etc.), uma vez que a análise aqui apresentada abarcou apenas o conhecimento produzido por dissertações de mestrado e teses de doutorado. Tal análise é necessária para compreender melhor de que maneira a investigação do comportamento verbal se estabeleceu e se expandiu pelo país.

\section{Referências}

Andery, M. A. P. A. (2001). Notas para uma revisão sobre comportamento verbal. In H. J. Guilhardi, M. B. B. P. Madi, P. B. Queiroz, \& M. C. Scoz (Eds.), Sobre comportamento e cognição: expondo a variabilidade (Vol. 7, pp. 372-385). Santo André, SP: ESETec.

Andery, M. A. P. A. (2010). Especificidade e implicações da interpretação da linguagem como comportamento verbal. In E. Z. Tourinho, \& S. V. Luna (Eds.), Análise do comportamento: investigações históricas, conceituais e aplicadas (pp. 61-101). São Paulo: Roca

Donahoe, T. W. (1998). Interpreting verbal behavior. The Analysis of Verbal Behavior, 15, 107-112.

Drash, P. W., \& Tudor, R. M. (1991). A standard methodology for the analysis, recording, and control of verbal behavior. The Analysis of Verbal Behavior, 9, 49-60.

Engelmann, A. (1969). Relatos verbais: um campo de investigação da psicologia (Unpublished master's thesis). Universidade de São Paulo, São Paulo.

Eshleman, J. W. (1991). Quantified trends in the history of verbal behavior research. The Analysis of Verbal Behavior, 9, 61-80. 
Fidalgo, A. P. (2011). O estudo do comportamento verbal no Brasil: uma análise com base em resumos de dissertações e teses (Unpublished master's thesis). Pontifícia Universidade Católica de São Paulo, São Paulo.

Hall, G. A. (1998). Promoting synthesis in the analysis of verbal relations. The Analysis of Verbal Behavior, 15, 113-116.

Hall, G. A., \& Chase, P. N. (1991). The relationship between stimulus equivalence and verbal behavior. The Analysis of Verbal Behavior, 9, 107-119.

Horne, P. J., \& Lowe, C. F. (1997). Toward a theory of verbal behavior. Journal of the Experimental Analysis of Behavior, 68, 271-296.

Leigland, S. (1998). The methodological challenge of the functional analysis of verbal behavior. The Analysis of Verbal Behavior, 15, 125-127.

Mendonça, M. M. (2010). Diferentes formas de regras no controle do comportamento (Unpublished master's thesis). Pontifícia Universidade Católica de São Paulo, São Paulo.

Micheletto, N., Guedes, M. C., César, G., \& Pereira, M. E. M. (2010). Disseminação do conhecimento em Análise do Comportamento produzido no Brasil (1962-2007). In E. Z. Tourinho, \& S. V. Luna (Eds.), Análise do Comportamento: investigações históricas, conceituais e aplicadas (pp. 101-125). São Paulo: Roca.

Micheletto, N., Guedes, M. C., Pereira, M. E. M., \& Silva, C. B. (2008). Banco de dados de dissertações e teses em Análise do Comportamento no Brasil 1968-2007 (BDTACB). Centro de Referências do Behaviorismo Radical do Laboratório de Estudos Históricos em Análise do Comportamento (LEHAC) do Programa de Estudos Pós-Graduados em Psicologia Experimental da PUC-SP.
Morris, E. K., Todd, J. T., Midgley, B. D., Schneider, S. M. \& Johnson, L. M. (1985). Conclusion: Some historiography of behavior analysis and some behavior analysis of historiography. In J. T. Todd, \& E. K. Morris (Eds.), Modern perspectives on B. F. Skinner and contemporary behaviorism (pp. 131-158). Westport: Greenwood Press.

Moroz, M., Rubano, D. R., Rodrigues, M. E., \& Lucci, M. A. (2001). Comportamento verbal: análise de produção científica nacional publicada no quinquênio 1994-1998. Revista Brasileira de Terapia Comportamental e Cognitiva, 3, 69-82.

Normand, M. P., Fossa, J. F., \& Poling, A. (2002). Publication trends in the analysis of verbal behavior: 1982-1998. The Analysis of Verbal Behavior, 17, 167-173.

Sidman, M., \& Tailby, W. (1982). Conditional discrimination vs. Matching to sample: an expansion of the testing paradigm. Journal of the Experimental Analysis of Behavior, 37, 5-22.

Skinner, B. F. (1969). An operant analysis of problem solving. In Contingencies of reinforcement: A theoretical analysis (pp. 133-171). New York: Appleton-Century-Crofts. (Trabalho original publicado em 1966)

Skinner, B. F. (1986). The evolution of verbal behavior. Journal of the Experimental Analysis of Behavior, 15, 115-122.

Skinner, B. F. (1992). Verbal behavior. Englewood Cliffs, NJ: Prentice Hall. (Trabalho original publicado em 1957)

Wulfert, E., Dougher, M. J., \& Greenway, D. E. (1991). Protocol analysis of the correspondence of verbal behavior and equivalence class formation. Journal of the Experimental Analysis of Behavior, 56, 489-504.

Recebido em 04.11.2013

Primeira decisão editorial em 10.01.2014

Versão final em 06.03.2014

Aceito em 27.03.2014 